Results Among 425 PHIV aged 13-19 $(n=409)$ and 20-24 ( $=16)$ at start of the 10-year period, $51(12 \%)$ were diagnosed with $\geq 1$ STI. Incidence was 1.3/100 person-years. There were 117 diagnoses among 51 PHIV; 25 (49\%) had an STI on 2 or more occasions. Incidence was significantly higher among females (2.1/100 personyears; $95 \%$ CI, $1.5-2.9)$ versus males $(0.7 / 100,000$ person-years; $95 \%$ CI, 0.4-1.1), and elevated among black PHIV persons (1.9/100 person-years; 95\% CI, 1.3-2.6). The PHIV case rate $(28,471 / 100,000$ persons) was $12 \%$ higher than the general population rate $(25,290 / 100,000)$.

Conclusions We documented substantial risk of STI among PHIV, which exceeds risk among other youth. True incidence may be higher than measured if PHIV had not initiated sexual activity as of our follow-up period. All sexually active youth should receive regular STI screening and education; because STI risk behaviours place partners at risk for HIV, PHIV should additionally get interventions around ARV adherence and HIV disclosure. PHIV in HIV care have unique opportunities for such prevention.

\section{P3.172 PREVALENCE OF GENITAL INFECTIONS WITH CHLAMYDIA TRACHOMATIS (CT), NEISSERIA GONORRHOEA (NG) AND TRICHOMONAS VAGINALIS (TV) IN CLIENTS OF PUBLIC HIV COUNSELLING INSTITUTIONS IN NORTH RHINE- WESTPHALIA, GERMANY: THE STI-HIT STUDY}

doi:10.1136/sextrans-2013-051184.0629

V Bremer, ${ }^{1} \mathrm{~K}$ Jansen, ${ }^{1} \mathrm{G}$ Steffen, ${ }^{1} \mathrm{~N}$ Sarma, ${ }^{2} \mathrm{~A}$ Lucht, ${ }^{2} \mathrm{D}$ Muenstermann, ${ }^{2} \mathrm{C}$ Tiemann. 'Robert Koch-Institut, Berlin, Germany; '2abor Krone, Bad Salzuflen, Germany

Background Chlamydia (CT), gonococci (NG) or trichomonas (TV) infections are not notifiable in Germany. Only few prevalence data exist for the general population. We measured CT, NG and TV prevalence in clients attending local public health authorities (LPHA) offering anonymous HIV tests to evaluate whether these tests should be routinely offered to this population.

Methods LPHA in North Rhine-Westphalia screened clients for genital infections with CT, NG and TV on basis of self-collected vaginal swabs (women) and self-collected urine samples (men), using Transcription-mediated amplification (CT and NG: APTIMA Combo $2^{\circledR}$; TV: APTIMA ${ }^{\circledR}$ Trichomonas vaginalis-Assay). We collected information on sociodemographics, sexual and HIV testing behaviour, as well as clinical symptoms.

Results Between 17.11.2012-05.02.2013, 448 men, 357 women and 4 transgender were enrolled by 18 LPHA. Median age was 30 years. $74.7 \%$ were born in Germany. Participants reported a median of 2 sexual partners within last six months. A total of $67.7 \%$ had a prior HIV test. Most frequent reasons for HIV test were sexual contact to person with unknown HIV status (57.0\%), new partner (28.9\%), and sex work (8.7\%). Overall, $18.6 \%$ had at least one prior STI. Thirty-one (3.8\%) persons tested positive for CT, $5(0.6 \%)$ for NG, and $2(0.2 \%)$ for TV; of those 4 NG and 2 TV infections were in sex workers. Prevalence for CT was highest in women $(4.5 \%)$ and sex workers $(5.7 \%)$. A total of $18.2 \%$ of STI-negative and $23.5 \%$ of STI-positive clients had clinical symptoms $(p=0.43)$.

Conclusions Persons attending LPHA for an HIV test do not seem to be a high risk group for STI. NG and TV prevalence is low in this population, except for sex workers. Routine CT testing should be offered to clients asking for an HIV test, independent of symptoms. Sex workers should also be screened for NG and TV.

\section{P3.173 STI SCREENING AND DIAGNOSIS HISTORY AMONG WOMEN WHO HAVE SEX WITH WOMEN AND MEN}

doi:10.1136/sextrans-2013-051184.0630

'V R Schick, 'A Bell, ' $\mathrm{C}$ Neal, 'B Van Der Pol, 'B Dodge, '2J D Fortenberry. 'Indiana University, Bloomington, IN, United States, 2Indiana University, Indianapolis, IN, United States
Background Research suggests that bisexually-identified women may be more likely to report an STI diagnosis within their lifetime than women who identify as heterosexual or homosexual. In the present study, the role of behaviour and identity were explored in relation to self-reported STI screening and diagnosis history.

Methods Behaviorally bisexual women (defined as genital contact with a male and female partner within the past 12 months) were recruited through websites, flyers, community events and participant referrals. Participants were asked to complete an on-line survey and an in-person interview. Participants self-reported details of sexual behaviours, sexual orientation/identity, STI screening and STI diagnosis history. They were also given the option to provide selfcollected vaginal, oral, and anal samples for STI screening.

Results Participants $(\mathrm{N}=80)$ ranged in age from 18 to 51 $(\mathrm{M}=26.74, \mathrm{SD}=7.97)$. Over three-quarters of the participants reported a history of STI screening. Over a quarter of the participants who reported screening reported a diagnosis of Chlamydia, HPV and/or bacterial vaginosis. Participants who identified as bisexual were more likely to report STI screening than their counterparts. The majority of participants provided a vaginal, oral, and/or anal sample for STI screening. None of the participants tested positive for Trichomoniasis or Gonorrhea with approximately $7 \%$ of participants testing positive for Chlamydia.

Conclusion The majority of participants reported STI screening with a sizable minority of participants reporting a positive STI diagnosis. The high proportion of participants who provided a vaginal, oral and/or anal sample indicates a high acceptability rate. The relationship of identity to STI screening in a sample of WSWM highlights the complexity of interventions targeted towards populations defined solely by identity or behaviour.

\section{P3.174 THE SEXUAL HEALTH OF MALE AND FEMALE SEX WORKERS ATTENDING GENITOURINARY MEDICINE CLINICS IN ENGLAND}

doi:10.1136/sextrans-2013-051184.0631

'L Mc Grath Lone, ${ }^{2} \mathrm{~K}$ Marsh, ${ }^{2} \mathrm{G}$ Hughes, 'H Ward. 'Imperial College, London, UK; ${ }^{2}$ Health Protection Agency, London, UK

Background Sex workers (SWs) are assumed to be at increased risk of sexually transmitted infections (STIs), but there are limited comparative data with other population groups. Previously, SW sexual health data were only available from special studies, but it is now gathered routinely as part of the Genitourinary Medicine Clinic Activity Dataset (GUMCAD), a national STI surveillance system. Methods Twelve months of GUMCAD data were analysed descriptively with the prevalence of STIs among SWs and other genitourinary (GUM) clinic attendees investigated. The increased risk of STI in SWs was estimated using logistic regression.

Results In 2011, 3,192 SWs (2,704 females, 488 males) were recorded as making 10,481 visits to GUM clinics. These visits were reported from a minority of clinics (primarily large, specialist centres in London). SWs utilised a variety of services including sexual health screens, HIV tests and vaccination; however, a minority of SWs were not recorded as having STI/HIV tests. Disparities in sexual health were observed among SWs. Male SWs had worse sexual health outcomes than female SWs (e.g. period prevalence of gonorrhoea among those tested: $17.6 \%$ vs. $2.7 \%$ ) and migrant female SWs had better outcomes than UK-born female SWs (e.g. period prevalence of chlamydia among those tested: $8.5 \%$ vs. $13.5 \%$ ). SWs also had an increased risk of STI when compared with other attendees, with the greatest risks in male SWs (Chlamydia: ORadj:3.98, 95\% CI: 3.05-5.18, p < 0.001).

Conclusions Routinely-gathered GUMCAD data can be used to assess the sexual health of SWs in England. Disparities in sexual health and differences in service utilisation appear to exist between 